\title{
ON A THEOREM OF VAN DER WAALS
}

\author{
BY PAUL, SAUREL.
}

Let us consider a chemical system of $r$ phases formed by means of $n$ independent components. Gibbs has shown ${ }^{\mathrm{T}}$ that when the system is in equilibrium there exists for each phase a relation of the form

$$
\begin{aligned}
v_{i} d \Pi=\eta_{i} d \mathrm{~T}+m_{i 1} d \mu_{i \mathrm{x}}+m_{i z} d \mu_{i \mathrm{z}}+\ldots+m_{i n} d \mu_{i n}, \\
i=\mathrm{I}, 2, \ldots, r,
\end{aligned}
$$

in which $\Pi$ and $\mathrm{T}$ denote the pressure and the temperature, $v_{i}$ and $\eta_{i}$ denote the volume and the entropy of the unit of mass of the $i$-th phase, $m_{i j}$ denotes the mass of the $j$-th component which is contained in the unit of mass of the $i$-th phase, and $\mu_{i j}$ denotes the chemical potential of the $j$-th component in the $i$-th phase.

Moreover, when the system is in equilibrium we have the relations

$$
\mu_{1 j}=\mu_{2 j}=\ldots=\mu_{r j}, \quad j=\mathrm{I}, 2, \ldots n,
$$

so that in a reversible cliange we have

$$
d \mu_{i j}=d \mu_{2 j}=\ldots=d \mu_{r j}, \quad j=\mathrm{I}, 2, \ldots n .
$$

Consider now a univariant system, that is to say a system for which $r=n+\mathrm{I}$. We can, in this case, eliminate from the $n+\mathrm{I}$ equations $\mathrm{I}$, the $n d \mu$ 's. The result of the elimination is ${ }^{2}$

$$
\left|\begin{array}{ccccc}
v_{1}, & m_{11}, & m_{12}, & \ldots, & m_{1 n} \\
v_{2}, & m_{21}, & m_{22}, & \ldots, & m_{2 n} \\
\vdots & \vdots & \vdots & & \vdots \\
v_{n+1}, & m_{n+1,1}, & m_{n+1,2}, & \ldots, & m_{n+\mathrm{I}, n}
\end{array}\right| d \mathrm{II}
$$

${ }^{1}$ On the Equilibrium of Heterogeneous Substances, p. I54.

2 On the Equilibrium of Heterogeneous Substances, p. I54. 


$$
=\left|\begin{array}{lcccc}
\eta_{1}, & m_{11}, & m_{12}, & \ldots, & m_{1 n} \\
\eta_{2}, & m_{21}, & m_{22}, & \ldots, & m_{2 n} \\
\vdots & \vdots & \vdots & & \vdots \\
\eta_{n+1}, & m_{n+\mathrm{I}, \mathrm{I}}, & m_{n+1,2}, & \ldots, & m_{n+1, n}
\end{array}\right| d \mathrm{~T} .
$$

This equation of Gibbs' will enable us to establish van der Waals' theorem. Let us consider first a two component univariant systenl ; equation 4 then becomes

$$
\frac{d \mathbf{I I}}{d v^{2}}\left|\begin{array}{lll}
v_{1}, & m_{11}, & m_{12} \\
v_{21}, & m_{21}, & m_{22} \\
v_{3}, & m_{31}, & m_{32}
\end{array}\right|=\left|\begin{array}{lll}
\eta_{1}, & m_{11}, & m_{12} \\
\eta_{2}, & m_{21}, & m_{22} \\
\eta_{31}, & m_{31}, & m_{32}
\end{array}\right| .
$$

If the concentrations of two of the phases, for example the phases $I$ and 2 , become equal, this equation may be written in the form:

$$
\frac{d \Pi}{d \mathrm{~T}}\left|\begin{array}{ccc}
v_{1}^{\prime}-v_{2}^{\prime}, & 0, & 0 \\
v_{2}, & m_{21}, & m_{22} \\
v_{31}, & m_{31}, & m_{32}
\end{array}\right|=\left|\begin{array}{ccc}
\eta_{1}-\eta_{2}, & 0, & 0 \\
\eta_{2}, & m_{21}, & m_{22} \\
\eta_{3}, & m_{i 1}, & m_{32}
\end{array}\right|,
$$

or, better,

$$
\left(v_{1}-v_{2}\right) \frac{d \mathrm{II}}{d \mathrm{~T}}\left|\begin{array}{ll}
m_{21}, & m_{22} \\
m_{31}, & m_{32}
\end{array}\right|==\left(\eta_{1}-\eta_{2}\right)\left|\begin{array}{ll}
m_{21}, & m_{22} \\
m_{31}, & m_{32}
\end{array}\right| .
$$

If the concentrations of the third phase are not equal to those of the first two, the determinant which appears in this equation is not equal to zero and we may write the equation in the form :

$$
\frac{d \pi}{d T}=\frac{\eta_{2}-\eta_{1}}{v_{2}-v_{1}}
$$

If the difference $v_{2}-v_{1}$ is very small in comparison with $\eta_{2}-\eta_{\mathrm{r}}, \frac{d \Pi}{d T}$ will be very large. In other words, the curve which represents the temperatures and the equilibrium pressures of the univariant system will have a tangent very nearly perpendicular to the axis of temperatures. Thus the temperature passes, in general, through a maximum or a minimum. 
This theorem; which is due to van der Waals, ${ }^{\mathrm{T}}$ has been verified experimentally by Roozeboom. ${ }^{2}$ A demonstration of the theorem has been given also by Duhem. ${ }^{3}$

We can go somewhat further. In a univariant system the variable concentrations are functions of the temperature or of the pressure. We may therefore write

$$
\frac{d m_{i j}}{d T}=\frac{d m_{i j}}{d \Pi \underline{\Pi}} \cdot \frac{d \Pi}{d T} .
$$

When the concentrations of the phases I and 2 become equal, $\frac{d \mathrm{n}}{d \mathrm{~T}}$ becomes very large; consequently, if

$$
\frac{d m_{i j}}{d \mathrm{II}} \neq 0
$$

$\frac{d m_{i j}}{d \mathrm{~T}}$ will be very large. Thus, when the concentrations of the two phases become equal, the curve which represents the relation between the temperature and a variable concentration $m_{i j}$ will have a tangent almost perpendicular to the axis of temperatures. This theorem which is also dne to van der Waals has been experimentally verified by Roozeboom.

The two theorems which we have just established can be extended without difficulty to a univariant system formed by means of more than two components. For, if the concentrations of the pliases I and 2 become equal, equation 4 can be written in the form

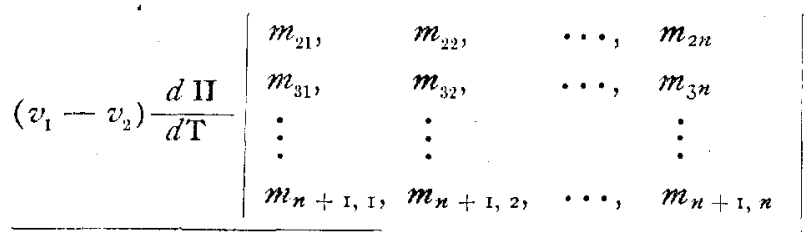

${ }^{1}$ Roozeboom. Sur les conditions d'équilibre de deux corps dans les trois états, solide, liquide et gazeux, d'après M. v. d. Waals. Recueil des Travaux chimiques des Pays-Bas, 5, 335 (1886). Roozeboom. Zeit. phys. Chem. 2, 463 (I 888 ).

${ }^{2}$ Recueil des 'Travaux chimiques des Pays-Bas, 4, 356 (I886). Zeit. phys. Chem. 2, 454 (1888).

${ }^{3}$ Dissolutions et Mélanges, deuxième Mémoire: les Propriétés physiques des Dissolutions; Travaux et Mémoires des Facultés de Lille, 3, No. I2, I2I, ( IS93). Traité élémentaire de Mécanique chimique, 3, 279. 


$$
=\left(\eta_{1}-\eta_{2}\right)\left|\begin{array}{cccc}
m_{21}, & m_{1: 2}, & \ldots, & m_{2 n} \\
m_{31}, & m_{1: 2,}, & \ldots, & m_{3 n} \\
\vdots & \vdots & & \vdots \\
m_{n+1,1}, & m_{n+1,2}, & \ldots, & m_{n+1, n}
\end{array}\right|
$$

If the deternininant which appears in this equation is not equal to zero, that is to say if the bivariant system which we obtain by suppressing the phase $I$ is not at an indifferent point, the last equation can be put into the form:

$$
\frac{d \Pi}{d \Gamma^{\top}}=\frac{\eta_{2}-\eta_{1}}{v_{2}-v_{1}}
$$

and we need only repeat the preceding demonstrations to establish van der Waals' theorems in the general case. 